\title{
Comparative effect of angiotensin converting enzyme inhibitor versus angiotensin II type I receptor blocker in acute myocardial infarction with non-obstructive coronary arteries; from the Korea Acute Myocardial Infarction Registry - National Institute of Health
}

Joon Ho Ahn ${ }^{1 *}$, Ju Yong Hyun ${ }^{1 *}$, Myung Ho Jeong ${ }^{1}$, Ju Han Kim ${ }^{1}$, Young Joon Hong ${ }^{1}$,

Doo Sun Sim ${ }^{1}$, Min Chul Kim ${ }^{1}$, Hun-Sik Park², Doo-Il Kim ${ }^{3}$, Seung-Ho Hur ${ }^{4}$, Seok Kyu $\mathrm{Oh}^{5}$, Youngkeun $\mathrm{Ahn}^{1}$; and other KAMIR-NIH Registry Investigators

\begin{abstract}
${ }^{1}$ Department of Cardiology, Chonnam National University Hospital, Gwangju, Korea ${ }^{2}$ Department of Cardiology, Kyungpuk National University Hospital, Daegu, Korea ${ }^{3}$ Department of Cardiology, Inje University Haeundae Paik Hospital, Busan, Korea ${ }^{4}$ Department of Cardiology, Keimyung University Dongsan Hospital, Daegu, Korea ${ }^{5}$ Department of Cardiology, Wonkwang University Hospital, Iksan, Korea
\end{abstract}

\begin{abstract}
Background: Selecting angiotensin converting enzyme inhibitor (ACEI) or angiotensin II type I receptor blocker (ARB) in patients diagnosed as acute myocardial infarction (AMI) with non-obstructive coronary arteries (MINOCA) is not established. The purpose of this study is to compare the clinical effect of ACEI vs. ARB in MINOCA patients.

Methods and results: A total of 273 patients between November 2011 to June 2015, diagnosed with MINOCA who were registered in the Korea Acute Myocardial Infarction Registry - National Institute of Health were enrolled. Patients were divided into ACEI $(n=112)$ and ARB groups $(n=161)$. The primary endpoint was cumulative incidence of major adverse cardiac events (MACE) defined as cardiac death, recurrent MI, any new revascularization during 2 years clinical follow-up. Secondary endpoint was heart failure requiring re-hospitalization. Propensity score matching analysis was done. The incidence of primary endpoint was similar (10.4\% vs. 15.6\%, HR: 0.65; 95\% CI: 0.29-1.47; $p=0.301$ ) among both groups. However, the incidence of recurrent MI was significantly lower in ACEI group compared to ARB group (2.1\% vs. $10.4 \%$, HR: 0.18, 95\% CI: 0.04-0.86; $p=0.031$ ).

Conclusions: In the present study, the risk and incidence of MACE was similar between ACEI and $A R B$ therapy in MINOCA patients. However, ACEI significantly reduced the risk of recurrent MI. Further larger scale multi-center randomized clinical trials are needed to clarify the proper use of renin-angiotensin-aldosterone system blocker in these patients. (Cardiol J 2021; 28, 5: 738-745)
\end{abstract}

Key words: non-obstructive coronary arteries, angiotensin converting enzyme inhibitor/ /angiotensin II type I receptor blocker, prognosis

Address for correspondence: Youngkeun Ahn, MD, PhD, FACC, FSCAI, Department of Cardiology, Cardiovascular Center, Chonnam National University Hospital, Institute of Molecular Medicine, BK21 Plus, Chonnam National University Medical School, 42 Jebong-ro, Dong-gu, Gwangju 61469, Korea, tel: 82-62-220-4764, fax: 82-62-224-4764, e-mail: cecilyk@hanmail.net Received: 31.03 .2020 Accepted: 21.07.2020 Early publication date: 28.09 .2020

*The first two authors contributed equally.

This article is available in open access under Creative Common Attribution-Non-Commercial-No Derivatives 4.0 International (CC BY-NC-ND 4.0) license, allowing to download articles and share them with others as long as they credit the authors and the publisher, but without permission to change them in any way or use them commercially. 


\section{Introduction}

Over the past decades, a remarkable evolution has occurred in the field of interventional and pharmacological modalities in acute myocardial infarction (AMI) [1]. Numerous coronary angiographic findings revealed that nearly $95 \%$ of AMI patients had obstructive coronary disease [2]. However, the remaining $5 \%$ and as many as $10 \%$ of AMI patients had no significant stenosis in their luminal coronary angiogram and these patients were coined as AMI with non-obstructive coronary arteries (MINOCA) [3]. Previous clinical studies demonstrated that MINOCA patients were younger and showed a higher portion of female patients compared to conventional patients with obstructive coronary artery disease (CAD) $[4,5]$. The prognosis of MINOCA patients were known to be favorable over those with conventional obstructive $\mathrm{CAD}$, however several recent reports demonstrated that the actual prognosis was not benign [6-8]. And yet, accurate evidence based therapeutic guidelines for these specific groups of patients is lacking [9]. Current guidelines strongly recommend (class I, level of evidence A) angiotensin converting enzyme inhibitor (ACEI) for MI patients with left ventricular ejection fraction (LVEF) of less than $40 \%$ or with symptoms of heart failure (HF) unless contraindicated $[1,10]$. Moreover, ACEI was encouraged for all ST-segment elevation MI patients without contraindications to their use (class IIa, level of evidence A). Guidelines also mentioned that the use of another renin-angiotensin-aldosterone (RAA) system blocker, angiotensin II type I receptor blocker (ARB) should be spared to patients who were intolerant to ACEI (class I, level of evidence $\mathrm{B})$. ARB is considered as an alternative choice. The main principle in MINOCA treatment is treating the underlying mechanism. However as previously mentioned, there are no evidence based therapeutic guidelines in treatment for MINOCA patients [11].

The purpose of the present study is to compare the clinical effect of ACEI and ARB in patients diagnosed as MINOCA in Korean AMI patients.

\section{Methods}

Among the 13,650 patients enrolled in Korea Acute Myocardial Infarction - National Institute of Health (KAMIR-NIH) between November 2011 to June 2015, 704 patients that showed insignificant stenosis $(<50 \%)$ in their initial coronary angiogram were selected. The KAMIR-NIH is a prospec-

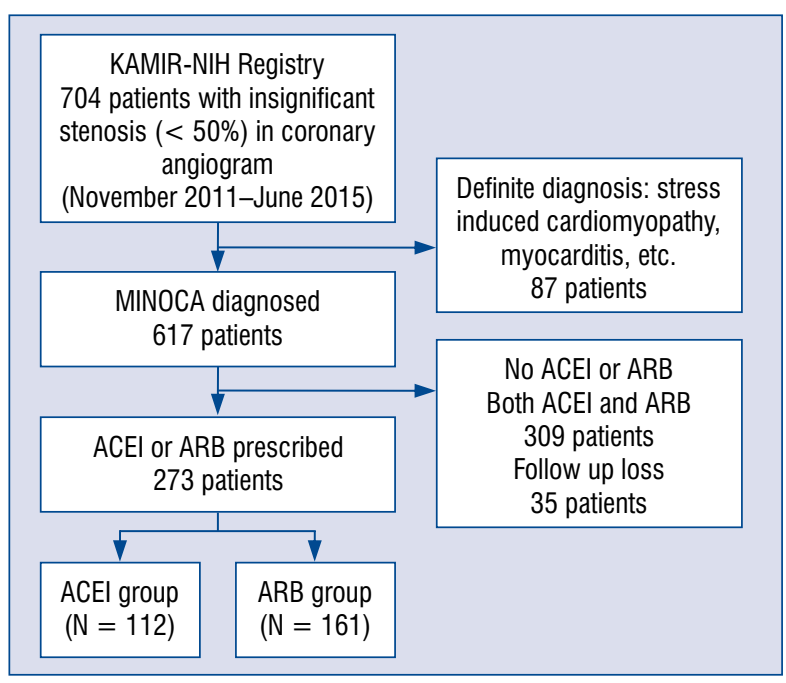

Figure 1. Study flow chart; ACEI - angiotensin converting enzyme inhibitor; $\mathrm{ARB}$ - angiotensin receptor blocker; MINOCA - myocardial infarction with non-obstructive coronary artery disease.

tive, open, multi-center, web-based cohort study to investigate the real-world outcome of Korean patients with AMI from 15 centers in Korea and has been supported by a grant of Korea Centers for Disease Control and Prevention since November 2011. Data were collected by trained study coordinators based on standardized protocol. Study protocol was approved by ethics committee at each participating institution. From these 704 individuals, the following patients were excluded sequentially: 87 patients with definite diagnosis such as stress induced cardiomyopathy or myocarditis, 309 patients who have not received either ACEI nor ARB or both, 35 patients who lacked outpatient follow-up data. A total of 273 patients were included in the present study. Patients were further divided into ACEI $(n=112)$ and ARB group $(n=161)$ (Fig. 1$)$. The current study was conducted according to the Declaration of Helsinki. The institutional review board of all participating centers approved the study protocol. The approval number was CNUH2011-172 of Chonnam National University Hospital. Written informed consent was obtained from all participating patients.

Blood samples for baseline laboratory tests were collected in the emergency room before diagnostic coronary angiography was carried out. Coronary angiography was performed by a standard technique through either radial or femoral artery. All patients received $300 \mathrm{mg}$ loading dose of acetylsalicylic acid (ASA) and 300-600 mg loading dose of 
clopidogrel before the coronary angiography unless contraindicated. During the hospital period, other medications including beta-blocker, statin, and calcium channel blocker (CCB) were also prescribed. Two-dimensional echocardiography was performed in all patients during the initial hospitalization period and LVEF was evaluated.

Diagnosis was AMI was made according to the clinical presentation, 12-lead electrocardiogram findings and change in cardiac biomarkers. Patients were categorized as MINOCA if the diagnosis of AMI was made and the coronary angiographic findings during initial hospitalization period showed no significant obstructive CAD (stenosis $<50 \%$ ) [9]. In the present study, the primary end point was the cumulative incidence of major adverse cardiac events (MACE) during 2 years clinical follow up. MACE was composite of cardiac death, recurrent MI, and any new revascularization. The secondary end point was HF requiring re-hospitalization. All deaths were considered cardiac deaths if non-cardiac deaths were excluded. Recurrent MI was defined as recurrent symptoms with new ST-segment elevation in electrocardiogram or re-elevation of cardiac markers to at least twice the upper limit of normal [12]. Any new revascularization was defined as interventional or surgical revascularization method including percutaneous coronary intervention or coronary artery bypass graft surgery.

\section{Statistical analysis}

The baseline clinical characteristics of both treatment group were analyzed. Continuous variables were presented as means \pm standard deviations and were compared by using unpaired the Student t-test or Mann-Whitney U test. Discrete variables were expressed as percentages and frequencies and were compared using chi-square statistics or the Fisher exact test. To minimize the selection bias in direct comparison between ACEI and ARB, propensity score analysis using multivariable logistic regression was done. Variables included were age, sex, atypical chest pain on admission, Killip class on admission, cardiovascular risk factors (hypertension, diabetes mellitus, dyslipidemia, smoking status), previous angina, previous MI, previous cerebrovascular disease, laboratory findings (serum glucose, renal function test, cardiac enzymes, lipid profiles), LVEF and prescribed medications (ASA, clopidogrel, beta-blocker, statin, and $\mathrm{CCB}$ ). Patients receiving ARB were matched 1 on 1 to the patients receiving ACEI with the nearest neighbor matching method (caliper width of 0.2 of the standard deviation). In the propensity score matched populations, the baseline characteristics were also analyzed. The risk of each clinical end point in both matched groups were compared by using the Cox proportional hazard regression model with covariables that showed statistical significance $(\mathrm{p}<0.1)$ in univariable analysis or were considered clinically important in the multivariate model. Hazard ratio (HR) with 95\% confidence interval (CI) were calculated.

All analyses were performed by using SPSS for Windows, version 25.0 (Armonk, NY). All statistical tests were 2 -tailed with statistical significance defined as a $\mathrm{p}$ value $\leq 0.05$.

\section{Results}

In the crude population, mean age of the both groups were $66.5 \pm 13.3$ years (ACEI group) and $68.0 \pm 10.9$ years (ARB group). ACEI group patients had a higher percentage of smokers ( $57.1 \%$ vs. $34.8 \% ; \mathrm{p} \leq 0.001)$ and tended to have a wider history of previous angina (23.2\% vs. $13.7 \%$; $\mathrm{p}=0.041)$. On the other hand, the ARB patient group had a higher portion of female patients $(37.5 \%$ vs. $54.7 \%$; $\mathrm{p}=0.005)$ and had more medical history of hypertension $(50.0 \%$ vs. $64.0 \%$; $\mathrm{p}=0.021$ ). Estimated LVEF was significantly lower in the ACEI group compared to the ARB group $(49.9 \pm 13.4$ vs. $55.5 \pm 12.4 ; \mathrm{p} \leq 0.001)$. After propensity score matching, 96 matched pairs of patients were selected and there was no difference in baseline clinical characteristics in both the ACEI and ARB groups (Table 1).

During the 2 year clinical follow-up period, cumulative incidence of primary end point MACE was similar between the ACEI and ARB groups in the crude population (10.4\% vs. $15.6 \%$; $\mathrm{p}=0.449$ ). Also, no difference was observed in the incidence of cardiac death $(7.1 \%$ vs. $4.3 \%$; $\mathrm{p}=0.561)$, recurrent MI ( $1.8 \%$ vs. $6.8 \% ; \mathrm{p}=0.054)$, any new revascularizations $(0.9 \%$ vs. $5.0 \%$; $\mathrm{p}=0.064)$ and HF requiring re-hospitalization $(5.2 \%$ vs. $7.3 \%$, HR: $0.42,95 \%$ CI: $0.11-1.68, \mathrm{p}=0.220)$. After propensity score matching analysis, the incidence and risk of recurrent MI was significantly lower in the ACEI group compared to the ARB group (2.1\% vs. $10.4 \%$, HR: $0.18,95 \%$ CI: $0.04-0.86, \mathrm{p}=0.031$ ) (Table 2). Other independent clinical factors for 2 years MACE were female sex (HR: 3.15, 95\% CI: 1.06-8.36; $\mathrm{p}=0.039)$ and estimated glomerular filtration rate $<60 \mathrm{~mL} / \mathrm{min} / 1.73 \mathrm{~m}^{2}$ (HR: 3.85 , 95\% CI: 1.36-9.89; $\mathrm{p}=0.011$ ) (Table 3). Kaplan-Meier curves for clinical outcomes are displayed in Figure 2. 
Table 1. Baseline clinical characteristics in crude and propensity score matched populations.

\begin{tabular}{|c|c|c|c|c|c|c|}
\hline & $\begin{array}{l}\text { ACEI group } \\
(n=112)\end{array}$ & $\begin{array}{l}\text { ARB group } \\
(n=161)\end{array}$ & $\mathbf{P}$ & $\begin{array}{l}\text { ACEl group } \\
(n=96)\end{array}$ & $\begin{array}{l}\text { ARB group } \\
(n=96)\end{array}$ & $\mathbf{P}$ \\
\hline Age [year] & $66.5 \pm 13.3$ & $68.0 \pm 10.9$ & 0.327 & $66.3 \pm 12.9$ & $66.6 \pm 11.7$ & 0.860 \\
\hline Female & $42(37.5 \%)$ & $88(54.7 \%)$ & 0.005 & $39(40.6 \%)$ & $39(40.6 \%)$ & 1.000 \\
\hline Atypical angina & $31(27.7 \%)$ & $48(29.8 \%)$ & 0.702 & $27(28.1 \%)$ & $22(22.9 \%)$ & 0.408 \\
\hline Killip class III, IV & $15(13.4 \%)$ & $11(6.8 \%)$ & 0.069 & $8(8.3 \%)$ & $9(9.4 \%)$ & 0.799 \\
\hline \multicolumn{7}{|l|}{ Risk factors: } \\
\hline Hypertension & $56(50.0 \%)$ & $103(64.0 \%)$ & 0.021 & $50(52.1 \%)$ & $54(56.3 \%)$ & 0.562 \\
\hline Diabetes mellitus & $30(26.8 \%)$ & $57(35.4 \%)$ & 0.133 & $28(29.2 \%)$ & $35(36.5 \%)$ & 0.282 \\
\hline Dyslipidemia & $10(8.9 \%)$ & $14(8.7 \%)$ & 0.947 & $9(9.4 \%)$ & $12(12.5 \%)$ & 0.488 \\
\hline Previous angina & $26(23.2 \%)$ & $22(13.7 \%)$ & 0.041 & $22(22.9 \%)$ & $18(18.8 \%)$ & 0.477 \\
\hline Previous MI & $28(25.0 \%)$ & $30(18.6 \%)$ & 0.206 & $23(24.0 \%)$ & $23(24.0 \%)$ & 1.000 \\
\hline Previous CVA & $7(6.3 \%)$ & $12(7.5 \%)$ & 0.701 & $7(7.3 \%)$ & $6(6.3 \%)$ & 0.774 \\
\hline Smoking & $64(57.1 \%)$ & $56(34.8 \%)$ & $<0.001$ & $50(52.1 \%)$ & $47(49.0 \%)$ & 0.665 \\
\hline \multicolumn{7}{|l|}{ Laboratory findings: } \\
\hline Serum glucose [mg/dL] & $151.9 \pm 74.9$ & $155.5 \pm 67.9$ & 0.686 & $153.0 \pm 75.4$ & $159.2 \pm 75.4$ & 0.569 \\
\hline eGFR $\left[\mathrm{mL} / \mathrm{min} / 1.7 \mathrm{~m}^{2}\right]$ & $79.8 \pm 29.4$ & $82.5 \pm 44.3$ & 0.573 & $81.9 \pm 28.9$ & $80.9 \pm 48.2$ & 0.864 \\
\hline CK-MB $[\mathrm{mg} / \mathrm{dL}]$ & $35.7 \pm 98.5$ & $24.7 \pm 41.9$ & 0.265 & $27.4 \pm 63.6$ & $29.8 \pm 50.8$ & 0.768 \\
\hline Troponin I [mg/dL] & $18.3 \pm 74.4$ & $7.8 \pm 17.2$ & 0.146 & $17.5 \pm 79.2$ & $9.7 \pm 19.4$ & 0.345 \\
\hline Total cholesterol [mg/dL] & $163.2 \pm 45.2$ & $164.4 \pm 43.9$ & 0.821 & $161.5 \pm 43.8$ & $168.3 \pm 50.8$ & 0.317 \\
\hline Triglyceride $[\mathrm{mg} / \mathrm{dL}]$ & $114.5 \pm 115.0$ & $118.9 \pm 85.1$ & 0.713 & $126.4 \pm 121.1$ & $131.9 \pm 99.7$ & 0.333 \\
\hline HDL cholesterol [mg/dL] & $50.3 \pm 29.7$ & $45.5 \pm 14.2$ & 0.074 & $47.7 \pm 14.5$ & $47.5 \pm 15.9$ & 0.937 \\
\hline LDL cholesterol [mg/dL] & $94.6 \pm 35.0$ & $99.9 \pm 31.9$ & 0.189 & $92.4 \pm 33.8$ & $95.1 \pm 36.1$ & 0.486 \\
\hline LVEF [\%] & $49.9 \pm 13.4$ & $55.5 \pm 12.4$ & 0.001 & $52.1 \pm 11.9$ & $52.3 \pm 12.5$ & 0.993 \\
\hline \multicolumn{7}{|l|}{ Medications: } \\
\hline ASA & $111(99.1 \%)$ & $158(98.1 \%)$ & 0.512 & $95(99.0 \%)$ & $94(97.9 \%)$ & 0.561 \\
\hline Clopidogrel & $102(91.1 \%)$ & $141(87.6 \%)$ & 0.364 & $87(90.6 \%)$ & $86(89.6 \%)$ & 0.809 \\
\hline Beta-blocker & $81(72.3 \%)$ & $108(67.1 \%)$ & 0.356 & $74(77.1 \%)$ & $68(70.8 \%)$ & 0.324 \\
\hline Calcium channel blocker & $33(29.5 \%)$ & $47(29.2 \%)$ & 0.961 & $28(29.2 \%)$ & $29(30.2 \%)$ & 0.874 \\
\hline Statin & $94(83.9 \%)$ & $141(87.6 \%)$ & 0.392 & $81(84.4 \%)$ & $86(89.6 \%)$ & 0.284 \\
\hline
\end{tabular}

Values are presented as the number (\%) of patients or mean standard deviation. ACEI — angiotensin converting enzyme inhibitor; ARB angiotensin receptor blocker; ASA - acetylsalicylic acid; CK-MB — creatine kinase-myocardial band isoenzyme; CVA - cerebrovascular accident; eGFR - estimated glomerular filtration rate; HDL — high density lipoprotein; LDL — low density lipoprotein; LVEF — left ventricular ejection fraction; $\mathrm{Ml}$ - myocardial infarction

Table 2. Clinical outcomes in crude and propensity matched populations.

\begin{tabular}{lccccccc}
\hline & $\begin{array}{c}\text { ACEI } \\
\text { group } \\
(\mathbf{n}=112)\end{array}$ & $\begin{array}{c}\text { ARB } \\
\text { group } \\
(\mathbf{n}=161)\end{array}$ & $\mathbf{P}$ & $\begin{array}{c}\text { ACEI } \\
\text { group } \\
(\mathbf{n}=96)\end{array}$ & $\begin{array}{c}\text { ARB } \\
\text { group } \\
(\mathbf{n}=96)\end{array}$ & $\begin{array}{c}\text { Adjusted HR } \\
(\mathbf{9 5} \% \mathbf{C l})\end{array}$ & $\mathbf{P}$ \\
\hline MACE* & $10(8.9 \%)$ & $19(11.8 \%)$ & 0.449 & $10(10.4 \%)$ & $15(15.6 \%)$ & $0.65(0.29-1.47 \%)$ & 0.301 \\
Cardiac death & $8(7.1 \%)$ & $7(4.3 \%)$ & 0.319 & $8(8.3 \%)$ & $6(6.2 \%)$ & $1.38(0.46-4.12 \%)$ & 0.561 \\
Recurrent MI & $2(1.8 \%)$ & $11(6.8 \%)$ & 0.054 & $2(2.1 \%)$ & $10(10.4 \%)$ & $0.18(0.04-0.86 \%)$ & 0.031 \\
Any new revascularization & $1(0.9 \%)$ & $8(5.0 \%)$ & 0.064 & $1(1.0 \%)$ & $5(5.2 \%)$ & $0.11(0.01-1.38 \%)$ & 0.085 \\
HF re-hospitalization & $7(6.3 \%)$ & $9(5.6 \%)$ & 0.819 & $5(5.2 \%)$ & $7(7.3 \%)$ & $0.42(0.29-1.47 \%)$ & 0.220 \\
\hline
\end{tabular}

${ }^{*}$ Composite of cardiac death, recurrent MI, and any new revascularization. Values are presented as $\mathrm{n}(\%)$ of patients or mean \pm standard deviation. $\mathrm{Cl}$ - confidence interval; HF — heart failure; HR - hazard ratio; MACE - major adverse cardiac event; other abbreviations as in Table 1. 
Table 3. Clinical predictors of major adverse cardiac event.

\begin{tabular}{lcc}
\hline & Adjusted HR (95\% CI) & P \\
\hline Female & $3.15(1.06-8.36)$ & 0.039 \\
ACEI & $0.79(0.32-1.97)$ & 0.613 \\
eGFR $<60 \mathrm{~mL} / \mathrm{min} / 1.73 \mathrm{~m}^{2}$ & $3.85(1.36-9.89)$ & 0.011 \\
\hline
\end{tabular}

Abbreviations as in Tables 1 and 2.

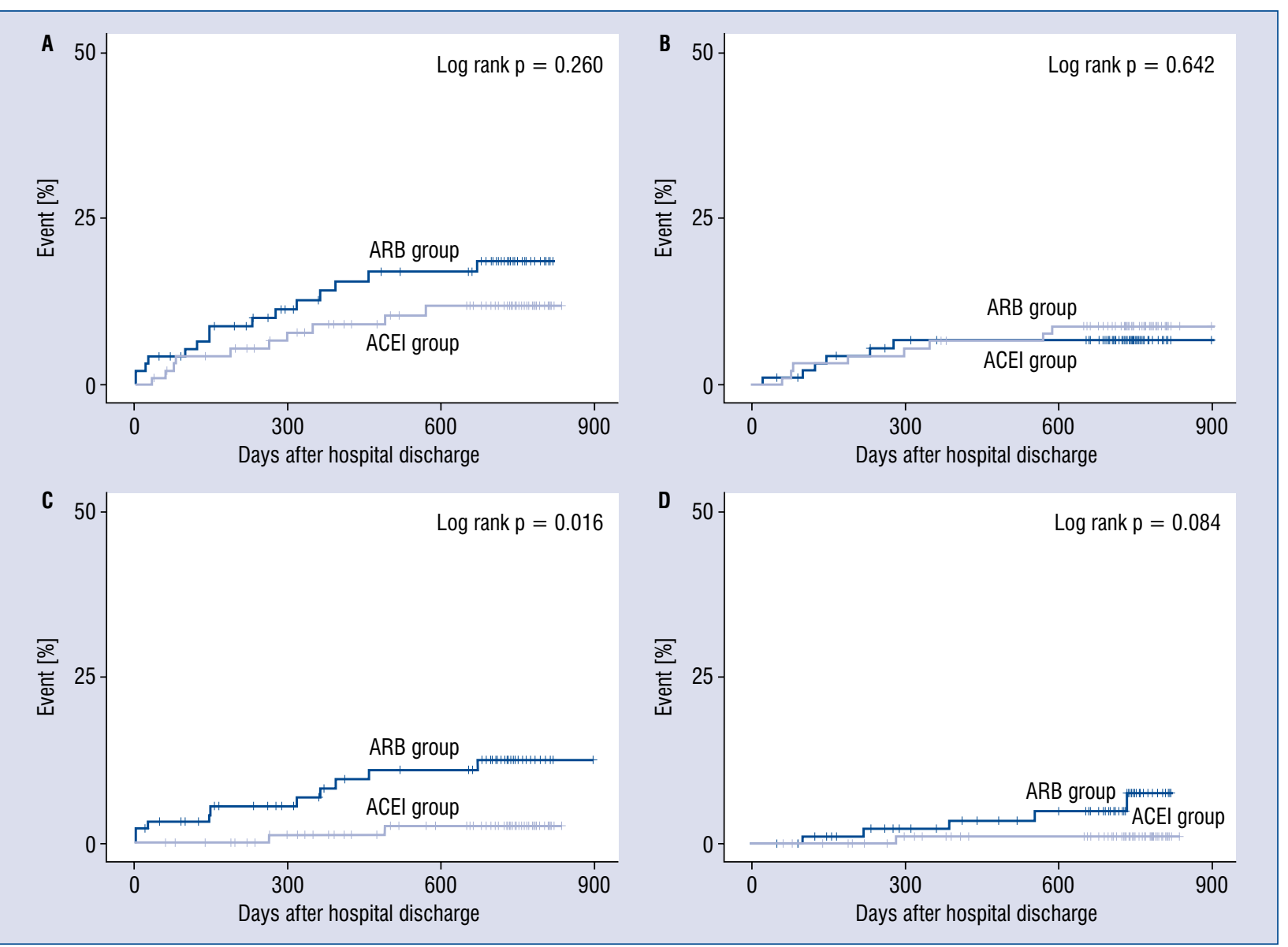

Figure 2. Kaplan-Meier curves for major adverse cardiac event (MACE) (A), cardiac death (B), recurrent myocardial infarction (MI) (C), any new revascularization (D); ACEI — angiotensin converting enzyme inhibitor; ARB — angiotensin receptor blocker.

\section{Discussion}

Results of the present study indicate that risk of MACE was similar in MINOCA patients treated with either ACEI or ARB. However, after propensity score matching analysis, it was revealed that ACEI therapy significantly lowered the risk of recurrent MI compared to the ARB therapy in MINOCA patients.
Early angiographic studies carried out by DeWood et al. [2] demonstrated that more than $90 \%$ of AMI patients show obstructive CAD $(\geq 50 \%$ stenosis) in their luminal coronary angiogram. Since then, there were emerging interests in the remaining $5-10 \%$ of the population group. Clinicians and interventional cardiologists defined this group of patients as MINOCA [9]. The proportion of MINOCA patients in the present study was $4.5 \%$, 
which was similar with the other previous reports. The following conditions should be included for a diagnostic approach to MINOCA: (i) Diagnostic criteria of AMI; (ii) No evidence of obstructive CAD ( $\geq 50 \%$ stenosis) in the initial coronary angiography; (iii) The overt cause of a specific diagnosis at the time of initial clinical presentation must be absent $[3,13]$.

MINOCA patients are known to be younger and showed a higher percentage of female patients compared to patients with conventional obstructive $\mathrm{CAD}[4,5]$. A recent meta-analysis reported that mean age of MINOCA patients was 58.8 years and the mean age of obstructive CAD patients was 61.2 years. $40 \%$ of MINOCA patients were women while only $24 \%$ were women in patients with obstructive CAD [5]. The proportion of female patients were $47 \%$ in the crude population and $40.6 \%$ in the propensity matched population in the present study, which was similar to meta-analysis data. However, our patient's mean age was older in both treatment groups (ACEI group: $66.5 \pm 13.3$ years, ARB group: $68.0 \pm 10.9$ years) compared to the meta-analysis data. Early studies revealed that MINOCA patients had better clinical outcomes compared to those with conventional obstructive CAD $[4,6]$. But, Kang et al. [14] showed that the incidence of 12 month MACE including all-cause death, MI and ischemic target vessel revascularization was $7.8 \%$ which was nearly the same as one vessel or two vessel obstructive CAD. The incidence of 2 years MACE in the present study was $13 \%$. This result could be considered to be higher than other results reported by Rossini et al. (9.1\%) [15]. The higher proportion of conventional cardiovascular risk factors such as smoking status and diabetes mellitus could have been the main contributing factor.

The clinical importance of MINOCA should not be underestimated because the proportion of MINOCA patients is not small and this group of patients are younger than conventional groups of patients with obstructive CAD. Nevertheless, the current clinical treatment guideline of MINOCA treatment is lacking. As previously mentioned, the mainstay of MINOCA therapy is based on treating the underlying pathophysiologic mechanism. The exact pathophysiologic mechanism of MINOCA needs further research but there are potential etiologic factors including plaque rupture or erosion, coronary artery spasm, coronary artery embolism or thrombus, microvascular dysfunction, etc. [11, 16, 17].

The benefit of long-term medical treatment in MINOCA patients was shown in several studies. An observational study of MINOCA patients in the
Swedish Web-system for Enhancement and Development of Evidence-based care in Heart disease Evaluated According to Recommended Therapy (SWEDEHEART) registry presented evidence of clinical benefit in long-term medical therapy for secondary prevention in MINOCA patients. During the mean follow up period of 4.1 years, there was an $18 \%$ risk reduction of primary outcomes in patients receiving RAA system blockers including ACEI or ARB [18]. However, there were no studies that directly compared the clinical effect of ACEI and ARB in MINOCA patients. According to available research, the current study is the first to directly compare the clinical effect between ACEI and ARB in MINOCA patients. The results showed that the incidence and risk of MACE in MINOCA patients were similar in the ACEI group and ARB group. The hard endpoint of cardiac death was similar in both treatment groups and this result was consistent with previous randomized clinical trials comparing ACEI and ARB [19, 20]. However, our results indicate that ACEI therapy lowered the risk of recurrent MI compared to the ARB therapy. These results could be inferred as a distinctive drug effect of ACEI by suppression of angiotensin II and bradykinin preservation and eventually resulted in restrained endothelial dysfunction [21,22]. Another potential cause might be the deleterious effect by overstimulation of angiotensin type II receptor by ARB which also could lead to an increase in angiotensin II levels [23]. Based on these factors, we considered that ACEI should be the first line treatment in MINOCA patients. Herein, we cautiously suggest that ACEI might be a preferable option in MINOCA patients because ACEI reduced the risk of recurrent MI compared to ARB.

\section{Limitations of the study}

The present study has several limitations. First, the study was not a randomized controlled clinical trial but a retrospective analysis based on a small sample sized population and selection bias might have existed. Although propensity score matching analysis was done and most potential confounders were adjusted for and analyzed, other variables that were associated with the clinical outcomes might not have been included in the present study. Second, the heterogeneity of MINOCA was not considered and the registry data lacks other diagnostic modalities that clarifies the accurate underlying cause of MINOCA including cardiac magnetic resonance (CMR) imaging or intravascular ultrasound, optical coherence tomography 
$[11,16]$. This was also the main shortcoming in the SWEDEHEART registry data and the they also stated that ideally the CMR proven myocarditis should have been excluded because one third of patients diagnosed as MI were actually discovered to have combined myocarditis in their CMR findings [18, 24]. An effort was made to select true MINOCA patients by excluding 87 patients diagnosed as stress induced cardiomyopathy or myocarditis however, a more precise and detailed study is needed because the future treatment guideline would be focused on MINOCA patients with unidentified cause. Third, accurate data reflecting the 2 year drug compliance of patients and specific categorization of ACEI and ARB and its prescribed dosage is lacking in the present study.

Further larger scale multi-center randomized clinical trials comparing the clinical effect of ACEI or ARB in MINOCA patients are needed for a proper RAA system blocking agent treatment in these groups of patients and to establish new treatment guidelines in MINOCA.

\section{Conclusions}

In the present study, the risk and incidence of MACE was similar between ACEI and ARB therapy in MINOCA patients. However, ACEI significantly reduced the risk of recurrent MI. Further larger scale multi-center randomized clinical trials are needed to clarify the proper use of RAA system blocker in these patients.

\section{Acknowledgments}

This research was supported by funding (2016-ER6304-02) of the Research of Korea Centers for Disease Control and Prevention, the National Research Foundation of Korea (NRF-2017M3A9E8023020) and the Chonnam National University Hospital Biomedical Research Institute (BCRI18017).

\section{Conflict of interest: None declared}

\section{References}

1. O'Gara PT, Kushner FG, Ascheim DD, et al. 2013 ACCF/AHA guideline for the management of ST-elevation myocardial infarction: executive summary: a report of the American College of Cardiology Foundation/American Heart Association Task Force on Practice Guidelines. Circulation. 2013; 127(4): 529-555.

2. DeWood MA, Spores J, Hensley GR, et al. Prevalence of total coronary occlusion during the early hours of transmural myocardial infarction. N Engl J Med. 1980; 303(16): 897-902, doi: 10.1056/NEJM198010163031601, indexed in Pubmed: 7412821.
3. Agewall S, Beltrame JF, Reynolds HR, et al. et al.. ESC working group position paper on myocardial infarction with non-obstructive coronary arteries. Eur Heart J. 2017; 38(3): 143-153.

4. Patel MR, Chen AY, Peterson ED, et al. Prevalence, predictors, and outcomes of patients with non-ST-segment elevation myocardial infarction and insignificant coronary artery disease: results from the Can Rapid risk stratification of Unstable angina patients Suppress ADverse outcomes with Early implementation of the ACC/AHA Guidelines (CRUSADE) initiative. Am Heart J. 2006; 152(4): 641-647, doi: 10.1016/j.ahj.2006.02.035, indexed in Pubmed: 16996828.

5. Pasupathy S, Air T, Dreyer RP, et al. Systematic review of patients presenting with suspected myocardial infarction and nonobstructive coronary arteries. Circulation. 2015; 131(10): 861-870, doi: 10.1161/CIRCULATIONAHA.114.011201, indexed in Pubmed: 25587100.

6. Larsen AI, Galbraith PD, Ghali WA, et al. Characteristics and outcomes of patients with acute myocardial infarction and angiographically normal coronary arteries. Am J Cardiol. 2005; 95(2): 261-263, doi: 10.1016/j.amjcard.2004.09.014, indexed in Pubmed: 15642564.

7. Safdar B, Spatz ES, Dreyer RP, et al. Presentation, clinical profile, and prognosis of young patients with myocardial infarction with nonobstructive coronary arteries (MINOCA): results from the VIRGO study. J Am Heart Assoc. 2018; 7(13), doi: 10.1161/ JAHA.118.009174, indexed in Pubmed: 29954744.

8. Planer D, Mehran R, Ohman EM, et al. Prognosis of patients with non-ST-segment-elevation myocardial infarction and nonobstructive coronary artery disease: propensity-matched analysis from the Acute Catheterization and Urgent Intervention Triage Strategy trial. Circ Cardiovasc Interv. 2014; 7(3): 285-293, doi: 10.1161/CIRCINTERVENTIONS.113.000606, indexed in Pubmed: 24847016.

9. Ibanez B, James S, Agewall S, et al. 2017 ESC Guidelines for the management of acute myocardial infarction in patients presenting with ST-segment elevation: The Task Force for the management of acute myocardial infarction in patients presenting with ST-segment elevation of the European Society of Cardiology (ESC). Eur Heart J. 2018; 39(2): 119-177.

10. Ponikowski P, Voors A, Anker S, et al. 2016 ESC Guidelines for the diagnosis and treatment of acute and chronic heart failure. Eur Heart J. 2016; 37(27): 2129-2200, doi: 10.1093/eurheartj/ ehw128.

11. Pasupathy S, Tavella R, Beltrame JF. Myocardial infarction with nonobstructive coronary arteries (MINOCA): the past, present, and future management. Circulation. 2017; 135(16): 1490-1493, doi: 10.1161/CIRCULATIONAHA.117.027666, indexed in Pubmed: 28416521.

12. Cutlip DE, Windecker S, Mehran R, et al. Academic Research Consortium. Clinical end points in coronary stent trials: a case for standardized definitions. Circulation. 2007; 115(17): 2344-2351.

13. Pasupathy S, Tavella R, Beltrame JF. The what, when, who, why, how and where of myocardial infarction with non-obstructive coronary arteries (MINOCA). Circ J. 2016; 80(1): 11-16, doi: 10.1253/circj.CJ-15-1096, indexed in Pubmed: 26597354.

14. Kang WY, Jeong MHo, Ahn YK, et al. Are patients with angiographically near-normal coronary arteries who present as acute myocardial infarction actually safe? Int J Cardiol. 2011; 146(2): 207-212, doi: 10.1016/j.jicard.2009.07.001, indexed in Pubmed: 19664828.

15. Rossini R, Capodanno D, Lettieri C, et al. Long-term outcomes of patients with acute coronary syndrome and nonobstructive coro- 
nary artery disease. Am J Cardiol. 2013; 112(2): 150-155, doi: 10.1016/j.amjcard.2013.03.006, indexed in Pubmed: 23602693.

16. Beltrame JF. Assessing patients with myocardial infarction and nonobstructed coronary arteries (MINOCA). J Intern Med. 2013; 273(2): 182-185, doi: 10.1111/j.1365-2796.2012.02591.x, indexed in Pubmed: 22998397.

17. Niccoli G, Scalone G, Crea F. Acute myocardial infarction with no obstructive coronary atherosclerosis: mechanisms and management. Eur Heart J. 2015; 36(8): 475-481, doi: 10.1093/eurheartj/ ehu469, indexed in Pubmed: 25526726.

18. Lindahl B, Baron T, Erlinge D, et al. Medical therapy for secondary prevention and long-term outcome in patients with myocardial infarction with nonobstructive coronary artery disease. Circulation. 2017; 135(16): 1481-1489, doi: 10.1161/CIRCULATIONAHA.116.026336, indexed in Pubmed: 28179398.

19. Pfeffer MA, McMurray JJ, Velazquez EJ, et al. Valsartan, captopril, or both in myocardial infarction complicated by heart failure, left ventricular dysfunction, or both. N Engl J Med. 2003; 349(20): 1893-1906.

20. Dickstein K, Kjekshus J. Effects of losartan and captopril on mortality and morbidity in high-risk patients after acute myocardial infarction: the OPTIMAAL randomised trial. Optimal
Trial in Myocardial Infarction with Angiotensin II Antagonist Losartan. Lancet. 2002; 360(9335): 752-760, doi: 10.1016/s01406736(02)09895-1, indexed in Pubmed: 12241832.

21. Strauss MH, Hall AS. The divergent cardiovascular effects of angiotensin converting enzyme inhibitors and angiotensin receptor blockers on myocardial infarction and death. Prog Cardiovasc Dis. 2016; 58(5): 473-482, doi: 10.1016/j.pcad.2015.11.004, indexed in Pubmed: 26586276.

22. Duchene J, Bader M. Bradykinin B2 receptor agonism: a novel therapeutic strategy for myocardial infarction? Am J Hypertens. 2010; 23(5): 459, doi: 10.1038/ajh.2010.32, indexed in Pubmed: 20404805.

23. Dézsi CA. Differences in the clinical effects of angiotensin-converting enzyme inhibitors and Angiotensin receptor blockers: critical review of the evidence. Am J Cardiovasc Drugs. 2014; 14(3): 167-173, doi: 10.1007/s40256-013-0058-8, indexed in Pubmed: 24385234 .

24. Tornvall P, Gerbaud E, Behaghel A, et al. Myocarditis or "true” infarction by cardiac magnetic resonance in patients with a clinical diagnosis of myocardial infarction without obstructive coronary disease: A meta-analysis of individual patient data. Atherosclerosis. 2015; 241(1): 87-91, doi: 10.1016/j.atherosclerosis.2015.04.816, indexed in Pubmed: 25967935. 